\title{
A Fatal Case of Infective Endocarditis Caused by an Unusual Suspect: Serratia marcescens
}

\author{
Yoshiro Hadano ${ }^{1,2}$, Toru Kamiya ${ }^{1,3}$ and Norimichi Uenishi ${ }^{1}$
}

\begin{abstract}
Serratia marcescens has been reported to cause infective endocarditis among intravenous drug users, but it is extremely rare in non-intravenous drug users in Japan. In this article, we report an 85-year-old woman with diabetes mellitus who presented with low-grade fever and general fatigue. She was administered intravenous prednisolone under a diagnosis of right Bell's palsy before this admission. Blood cultures revealed positive Serratia marcescens, which was complicated by multiple cerebral infarctions after admission. Transthoracic echocardiography on day 5 revealed vegetation on the mitral valve, which was diagnosed as infective endocarditis. An operation could not be performed because of the presence of multiple cerebral infarctions. She died on day 65 because of uncontrolled heart failure.
\end{abstract}

Key words: Serratia marcescens, infective endocarditis, non-HACEK

(Intern Med 51: 1425-1428, 2012)

(DOI: 10.2169/internalmedicine.51.6648)

\section{Introduction}

Serratia species are motile gram-negative bacilli and Serratia marcescens ( $S$. marcescens) is an important pathogen species of Serratia. S. marcescens causes nosocomial infections such as pneumonia, catheter associated urinary tract infection, septicaemia, meningitis, and wound infection $(1,2)$. Historically, S. marcescens has been reported to cause infective endocarditis among intravenous drug users $(3,4)$, but it is extremely rare in non-intravenous drug user (5). In this article, we report a fatal case of suspected healthcareassociated infective endocarditis caused by $S$. marcescens in a patient without a history of intravenous drug use.

\section{Case Report}

An 85-year-old Japanese woman with diabetes mellitus on insulin therapy and with hypertension was admitted to our hospital because of fever and general fatigue.

The patient had been well until three weeks earlier, when she developed right facial paralysis, including paralysis of her forehead, and was admitted to our hospital. She was ad- ministered prednisolone under a diagnosis of right Bell's palsy but her symptom persisted. One week later, she was discharged from our hospital. Three days after leaving the hospital, she developed a low-grade fever and general fatigue with some episodes of aspiration. One day prior to the second admission, she visited our emergency department and was admitted because of fever, chills and general fatigue. Her medications included an ACE inhibitor and intermediate-acting insulin. She did not use tobacco or illicit drugs and rarely drank alcohol. She denied any history of allergies.

On physical examination, her blood pressure was 132/68 $\mathrm{mmHg}$, pulse was 88 beats per minute, temperature $38.8^{\circ} \mathrm{C}$, the respiratory rate 20 breaths per minute, and the oxygen saturation $97 \%$ while she was breathing ambient air. Her eyes, nose, oral cavity, and throat were all normal. There was no palpable lymphadenopathy. The cardiovascular examination revealed a $2 / 6$ holosystolic murmur in the left fourth intercostal space radiating to the axilla. Neurologic examination was unremarkable except for right peripheral facial paralysis. No Osler's node, Janeway lesion, or petechiae were observed. The remainder of the examination was normal.

${ }^{1}$ Department of General Internal Medicine, Rakuwakai Otowa Hospital, Japan, ${ }^{2}$ Division of Infectious Diseases, Shizuoka Cancer Center, Japan and ${ }^{3}$ Division of Infectious Diseases, Rakuwakai Otowa Hospital, Japan

Received for publication October 3, 2011; Accepted for publication February 16, 2012

Correspondence to Dr. Yoshiro Hadano, hatayoshiyoshi@yahoo.co.jp 


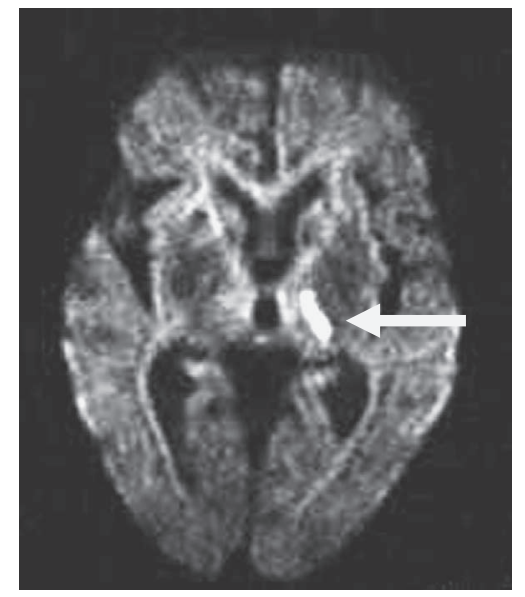

Figure 1. Diffusion-weighted image of the brain MRI with high intensity areas on the posterior limb of left internal capsule (arrow).

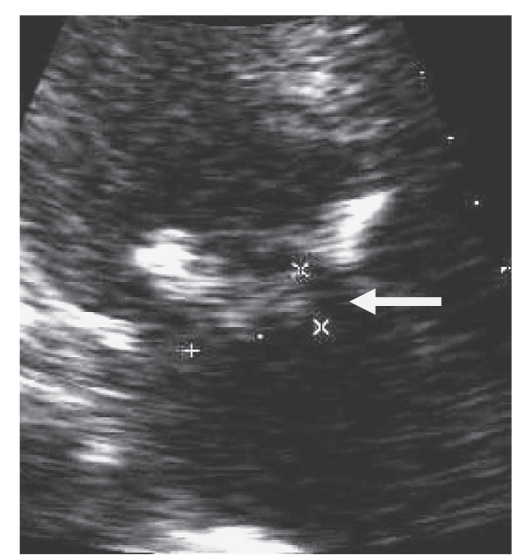

Figure 2. Transthoracic echocardiogram on day 5 with mitral valve vegetation (arrow).

Laboratory data obtained on the second admission revealed a white blood cell (WBC) count of $13,900 / \mu \mathrm{L}$ with $87 \%$ neutrophils, $11 \%$ lymphocytes, and $2 \%$ monocytes. Hemoglobin was $11.8 \mathrm{mg} / \mathrm{dL}$ with an MCV of $97.6 \mathrm{fL}$. Platelet count was $142,000 / \mu \mathrm{L}$. Erythrocyte sedimentation rate was $69 \mathrm{~mm} /$ hour. Serum chemistries revealed sodium of 130 $\mathrm{mEq} / \mathrm{L}$, potassium $3.5 \mathrm{mEq} / \mathrm{L}$, chloride $96 \mathrm{mEq} / \mathrm{L}$, blood urea nitrogen (BUN) $13.3 \mathrm{mg} / \mathrm{dL}$, creatinine $1.1 \mathrm{mg} / \mathrm{dL}$, glucose $219 \mathrm{mg} / \mathrm{dL}$, albumin $2.3 \mathrm{~g} / \mathrm{dL}$, total protein $5.5 \mathrm{~g} / \mathrm{dL}$, AST $32 \mathrm{IU} / \mathrm{L}$, ALT $19 \mathrm{IU} / \mathrm{L}$, total bilirubin $0.5 \mathrm{mg} / \mathrm{dL}, \mathrm{LDH}$ $256 \mathrm{IU} / \mathrm{L}$, CRP $6.3 \mathrm{mg} / \mathrm{dL}$, and HbA1c 6.0\%. Her urinalysis was normal. Chest radiography revealed no pneumonia, and electrocardiogram (ECG) showed complete right bundle branch block.

On hospital day 1, given recent episodes of aspiration, the patient was started on intravenous ampicillin-sulbactam. On day 2 , she suddenly developed a right hemiplegia and dysarthria. Diffusion-weighted imaging of the brain via magnetic resonance imaging showed multiple acute cerebral infarctions at the posterior limb of the left internal capsule and cerebellar hemisphere (Fig. 1). Her blood culture became positive for gram-negative bacilli, and contrast-enhanced ab- dominal computed tomography was performed to look for other embolic lesions and identify splenic infarction. Transthoracic echocardiography detected mild aortic regurgitation. Gram-negative bacilli was treated with intravenous gentamicin. On day 3, the blood culture grew S. marcescens. The sputum culture only grew normal oral flora and her urine culture was negative. The $S$. marcescens isolate was susceptible to piperacillin, ceftriaxone, ceftazidime, cefepime, meropenem, gentamicin, and levofloxacin. From day 3 , intravenous ceftazidime plus gentamicin were administered. On day 4, the follow-up blood cultures grew $S$. marcescens again. On day 5, she developed dyspnea suggestive of heart failure. Transthoracic echocardiography was repeated which revealed a $20 \times 10 \mathrm{~mm}$ floppy vegetation on the mitral valve with severe mitral regurgitation (Fig. 2). Her rheumatoid factor was positive and she was diagnosed as infective endocarditis due to $S$. marcescens. The second follow-up blood culture on day 5 was negative. On day 7 , gentamicin was stopped because of acute renal failure, but ceftadizime monotherapy was continued until day 42 .

Although an operation was considered, acute multiple cerebral infarction contraindicated her from undergoing the operation. Unfortunately, she died on day 65 because of uncontrolled heart failure.

\section{Discussion}

S. marcescens, a motile gram-negative bacillus classified as a member of the Enterobacteriaceae, is widespread in the environment but not a common microbial member of the fecal flora. Serratia species are occasionally recognized as causes of hospital-acquired infection, such as urinary tract infections, respiratory tract infections, and wound infections $(1,2)$.

With respect to infective endocarditis, Serratia species is quite rarely a causative organism. Analysis of 2,671 patients with definitive endocarditis from 2000 to 2005, nonHACEK group (species other than hemophilus species, Actinobacillus actinomycetemcomitans, Cardiobacterium hominis, Eikenella corrodens, or Kingella species) gram-negative bacilli was only $49(1.8 \%)$ and Serratia species was only 4 (0.14) (5). It also has been reported that patients who were recipients of presthtic devices and patients with cirrhosis appear to be at increased risk of gram-negative bacilli endocarditis $(5,6)$. In endocarditis due to Serratia species, $S$. marcescens is the most common organism, but $S$. liquefaciens has also been reported as a cause of endocarditis (7). As in the present case, left-sided endocarditis due to $S$. marcescens is usually refractory to medical therapy alone and uncontrolled congestive heart failure is a relatively frequent complication in this type of infection. The mortality rate of left-sided endocarditis due to Serratia species in patients who received only medical treatment is about eighty-five percent (7). Therefore, it is recommended that patients with left-sided endocarditis due to Serratia species undergo surgical replacement of the involved valve combined with antibi- 

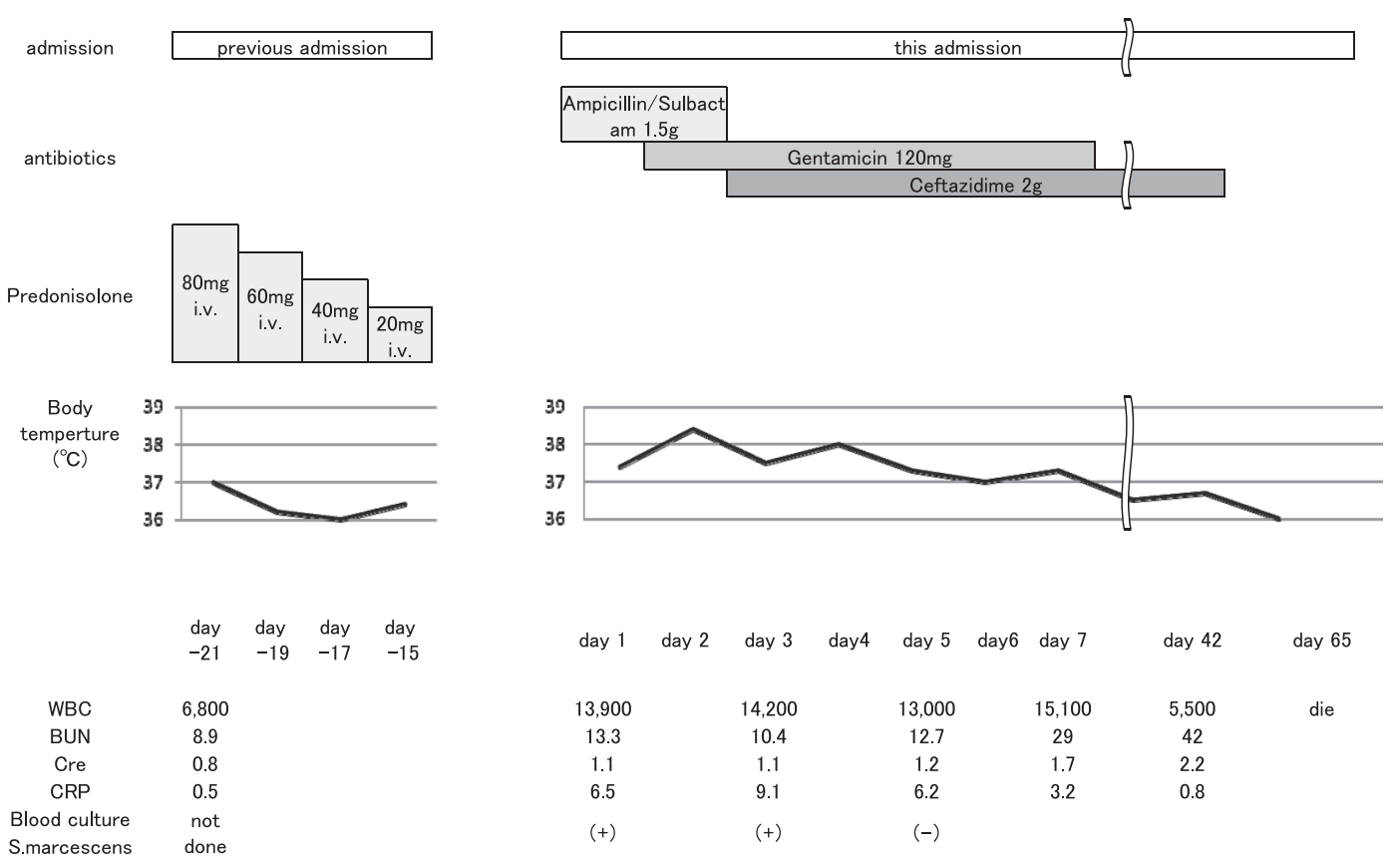

Figure 3. Clinical course.

otic treatment in the first few days. In the present case, indication for surgery was satisfied in terms of severe heart failure and large vegetation ( $>10 \mathrm{~mm}$ in diameter) but we could not perform an operation because of acute multiple cerebral infarction. On the other hand, right-sided endocarditis due to S. marcescens has been reported to be cured by antibiotics with or without surgery according to the reports in the 1970's $(3,4)$. Recent data showed that all patient of infectious endocarditis due to $S$. marcescens survived though a combination of antibiotics and early surgical treatment (5). Serratia species has been occasionally recognized as the cause of healthcare-associated bacteremia due to the contamination of blood products (8), cleaning solutions (9), and inadequate sterile techniques with intravenous medication $(10,11)$, however, a recent population-based study of Serratia species infection, including bacteremia in Canada, showed that $65 \%$ of infections were community-acquired infections (12) and similarly reports from Australia revealed that $47 \%$ of bacteremia episodes were started in the community (13). In the present case, bacteremia due to S. marcescens could have been caused by healthcare-associated infection during the first admission when she received intravenous fluid therapy. Although no outbreaks due to Serratia species occurred at that time, we believe that the cause of bacteremia was healthcare associated because there was no evidence of any entry without peripheral catheter associated infection.

In general, non-HACEK organisms including S. marcescens are infrequently a causative pathogen of infective endocarditis. This is probably due to their high sensitivity to serum bactericidal activity and low propensity to adhere to damaged endothelium (14). We suggest that the host immune status is important. Aubron et al. reported an higher incidence of Enterobacteriaceae endocarditis in immunocom- promised patients (15) and Durante-Mangoni et al. reported that diabetes mellitus was the major predisposing factor in elderly patients of infective endocarditis (16). The present patient had diabetes mellitus and steroid therapy was administered due to the initial diagnosis of Bell's palsy. We suggest the patient developed infective endocarditis caused by S. marcescens because the patient was older and likely immunocompromised.

In conclusion, we report a fatal case of infective endocarditis caused by $S$. marcescens complicated by multiple embolization in a patient without a history of intravenous drug use. It was probably caused by secondary healthcareassociated bacteremia. It is important to keep in mind that fatal infective endocarditis due to $S$. marcescens is rare, but can occur in elderly and/or immunocompromised patients.

\section{The authors state that they have no Conflict of Interest (COI).}

\section{Acknowledgement}

We are grateful to Dr. Sanjay Saint for his critical proofreading of the English manuscript.

\section{References}

1. Hejazi A, Falkiner FR. Serratia marcescens. J Med Microbiol 46: 903-912, 1997.

2. Acar JF. Serratia marcescens infections. Infect Control 7: 273278, 1986.

3. Mills J, Drew D. Serratia marcescens endocarditis: a regional illness associated with intravenous drug abuse. Ann Intern Med 84: 29-35, 1976.

4. Cooper R, Mills J. Serratia endocarditis. A follow-up report. Arch Intern Med 140: 199-202, 1980.

5. Morpeth S, Murdoch D, Cabell CH, et al. International Collaboration on Endocarditis Prospective Cohort Study (ICE-PCS) Investigators. Non-HACEK gram-negative bacillus endocarditis. Ann In- 
tern Med 147: 829-835, 2007.

6. Snyder N, Atterbury CE, Pinto Correia J, Conn HO. Increased concurrence of cirrhosis and bacterial endocarditis. A clinical and postmortem study. Gastroenterology 73: 1107-1113, 1977.

7. Chuang TY, Chuang CP, Cheng HH, Hsueh PR. Aortic valve infective endocarditis caused by Serratia liquefaciens. J Infect $\mathbf{5 4}$ 161-163, 2007.

8. Vonberg RP, Gastmeier P. Hospital-acquired infections related to contaminated substances. J Hosp Infect 65: 15-23, 2007.

9. Sartor C, Jacomo V, Duvivier C, Tissot-Dupont H, Sambuc R, Drancourt M. Nosocomial Serratia marcescens infections associated with extrinsic contamination of a liquid nonmedicated soap. Infect Control Hosp Epidemiol 21: 196-199, 2000.

10. Sunenshine RH, Tan ET, Terashita DM, et al. A multistate outbreak of Serratia marcescens bloodstream infection associated with contaminated intravenous magnesium sulfate from a compounding pharmacy. Clin Infect Dis 45: 527-533, 2007.

11. Pan A, Dolcetti L, Barosi C, Carnevale G, et al. An outbreak of Serratia marcescens bloodstream infections associated with misuse of drug vials in a surgical ward. Infect Control Hosp Epidemiol 27: 79-82, 2006.

12. Laupland KB, Parkins MD, Gregson DB, Church DL, Ross T, Pitout JD. Population-based laboratory surveillance for Serratia species isolates in a large Canadian health region. Eur J Clin Microbiol Infect Dis 27: 89-95, 2008.

13. Engel HJ, Collignon PJ, Whiting PT, Kennedy KJ. Serratia sp. bacteremia in Canberra, Australia. A population-based study over 10 years. Eur J Clin Microbiol Infect Dis 28: 821-824, 2009.

14. Brouqui P, Raoult D. Endocarditis due to rare and fastidious bacteria. Clin Microbiol Rev 14: 177-207, 2001.

15. Aubron C, Charpentier J, Trouillet JL, et al. Native-valve infective endocarditis caused by Enterobacteriaceae: report on 9 cases and literature review. Scand J Infect Dis 38: 873-881, 2006.

16. Durante-Mangoni E, Bradley S, Selton-Suty C, et al. Current features of infective endocarditis in elderly patients: results of the International Collaboration on Endocarditis Prospective Cohort Study. Arch Intern Med 168: 2095-2103, 2008.

(C) 2012 The Japanese Society of Internal Medicine http://www.naika.or.jp/imindex.html 